\title{
The Effectiveness of Afatinib in a Patient with Advanced Lung Adenocarcinoma Harboring Rare G719X and S768I Mutations
}

\author{
Masahiro Watanabe, Satoshi Oizumi, Shizuka Kiuchi, Noriyuki Yamada, \\ Hiroshi Yokouchi, Shinichi Fukumoto and Masao Harada
}

\begin{abstract}
:
The uncommon mutations in the EGFR (the epithelial growth factor receptor) gene include a heterogeneous group of genomic alterations within exons 18-21. The clinical response of patients with such mutations to EGFR tyrosine kinase inhibitor (EGFR-TKI) treatment, however, remains unclear. We herein report a case of advanced lung adenocarcinoma harboring complex exon 18 G719X (Gly719Xaa) and exon 20 S768I (Ser768Ile) mutations. The patient started to receive afatinib and has exhibited good response without progression for 12 months. Second-generation EGFR-TKIs might be an optimal treatment option for non-small cell lung cancers harboring these types of rare EGFR mutation.
\end{abstract}

Key words: non-small cell lung cancer, uncommon EGFR mutation, second-generation TKI, afatinib

(Intern Med 57: 993-996, 2018)

(DOI: 10.2169/internalmedicine.9565-17)

\section{Introduction}

Numerous studies have demonstrated the superiority of first-line epidermal growth factor receptor (EGFR) tyrosine kinase inhibitors (TKIs) over standard chemotherapy in regard to progression-free survival (PFS) and the response to treatment in non-small cell lung cancers (NSCLCs) with sensitive EGFR mutations. Thus, EGFR-TKI is currently the mainstay first-line systemic therapy for advanced EGFRmutant NSCLC.

The two most common EGFR mutations, exon 19 deletion (del19) and the Leu858Arg point mutation in exon 21 (L858R), account for $90 \%$ of all mutations in this gene. The remaining $10 \%$ of EGFR mutations (so-called uncommon mutations), are a heterogeneous group of genomic alterations that occur within exons 18-21.

Recently, the EGFR-TKI afatinib has been reported to show anti-tumor activity in patients with NSCLC harboring such uncommon EGFR mutations (1). We herein report a case of NSCLC harboring rare complex exon 18 (G719X) and exon 20 (S768I) mutations, which responded well to afatinib monotherapy.

\section{Case Report}

A 72-year-old female patient was referred to our hospital due to continuous cough and sputum. Routine laboratory examinations revealed high levels of serum CYFRA (4.4 ng/ $\mathrm{mL}$ ) and SLX (88.1 U/mL). Chest computed tomography revealed a primary lung tumor in the right S1a and an irregular, thickened interlobular septum and bronchovascular interstitium in the right upper and middle lobes (Fig. 1), which were considered to be primary lung cancer and carcinomatous lymphangitis, respectively. Clinical cT4N3M0 stage IIIB (UICC TNM Classification, 7th Edition) adenocarcinoma of the lung was diagnosed on the basis of a bronchoscopic tumor biopsy; there was no evidence of distant metastasis.

Complex G719X (Gly719Xaa) and S768I (Ser768Ile) mutations were detected in cytological samples by EGFR mutation testing. Based on recent reports demonstrating the antitumor activity of afatinib (a second-generation EGFR-TKI) in patients with uncommon EGFR mutations, we recom- 


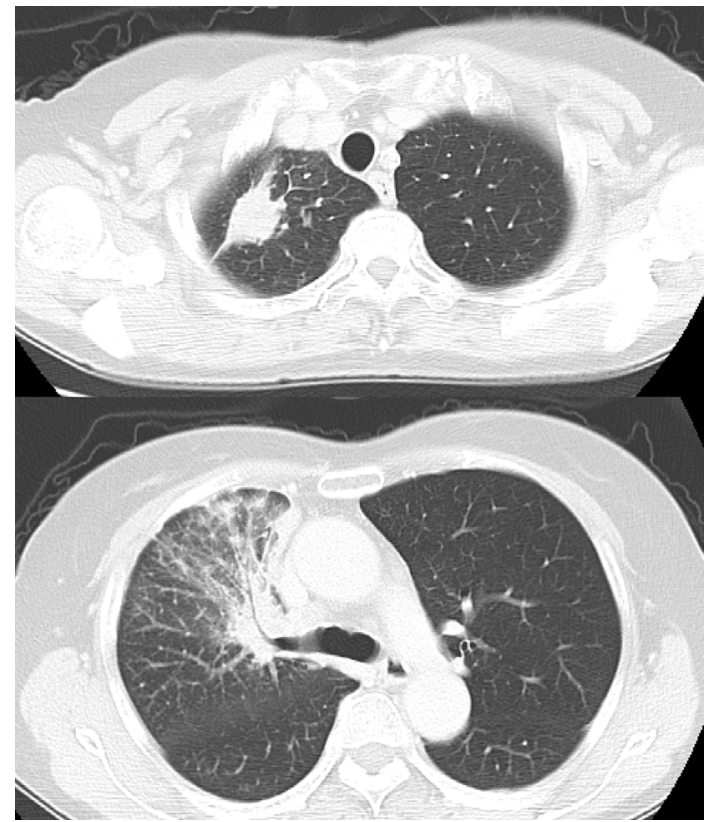

Figure 1. Chest computed tomography scans prior to afatinib treatment, showing a primary lung tumor in the right S1a, and an irregular, thickened interlobular septum and bronchovascular interstitium in the right upper lobe.

mended this therapy to the patient. After obtaining her consent, the patient was started on afatinib (40 mg/day); however serious adverse events, including diarrhea (Grade 2 according to the National Cancer Institute Common Terminology Criteria for Adverse Events 4.0), rash (Grade 2), stomatitis (Grade 2), and nausea (Grade 3) necessitated the temporary cessation of treatment. Subsequently, the afatinib dosage was reduced twice to relieve adverse events, and the patient currently receives a dose of $20 \mathrm{mg} /$ day (Fig. 2), which has resulted in substantial tumor shrinkage (Fig. 3). At 12 months since the start of afatinib treatment, no progressive disease has been observed in the patient.

\section{Discussion}

Generally, NSCLC patients with common EGFR mutations (such as del19 or L858R in exon 21) exhibit a dramatic response to EGFR-TKIs. Other mutations in exons 1821 , including complex mutations, are considered rare and the clinical response of such patients to EGFR-TKI treatment has remained unclear. Complex G719X and S768I mutations, which were detected in the current case, have previously been reported to be rare in the combined analysis of the LUX-Lung 2, LUX-Lung 3, and LUX-Lung 6 NSCLC cohorts (NCT00525148, NCT00949650, and NCT01121393, respectively); only five patients with these mutations were found among 600 afatinib-treated EGFR-mutant NSCLC cases $(5 / 600$ cases, $0.83 \%)$ (1). Among the uncommon mutations that were documented in the same analysis $(n=75$ patients), G719X and S768I mutations only represented $6.6 \%$ of the rare variants (5/75 cases) (1). In another report, com- plex G719X and S768I mutations were detected in 10/161 cases with uncommon EGFR mutations (6.2\%) (2).

To date, several studies have reported the distinct clinical effectiveness of first-generation EGFR-TKIs in the treatment of NSCLC cases involving uncommon mutations (2-6). The post-hoc analysis of NEJ002 (UMIN C000000376) demonstrated that the overall survival and PFS of gefitinib-treated patients with uncommon EGFR mutations (G719X and L861Q) were shorter in comparison to those with common mutations (6). Among the 10 NSCLC cases with G719X and S768I mutations, five patients exhibited partial responses to EGFR-TKI, whereas the other five cases developed resistance to therapy. Intriguingly, patients with complex uncommon EGFR mutations (G719X and L861Q, or G719X and S768I) had significantly longer PFS in comparison to patients with a single uncommon mutation (2). With respect to the individual mutations observed in the current case, first-generation EGFR-TKIs are considered to be moderately effective in patients with G719X (4), while S768I has been associated with a poor response to first generation EGFR-TKIs, especially gefitinib (7).

In preclinical experiments, Kobayashi et al. showed that EGFR exon 18 mutations are associated with unique sensitivity to afatinib and neratinib (second-generation EGFRTKIs), in comparison to first- and third-generation TKIs (8), suggesting that lung cancer patients with exon 18 mutations could be good candidates for treatment with afatinib. In addition, Banno et al. demonstrated that stably transfected $\mathrm{Ba}$ / F3 cell lines harboring an S768I mutation were sensitive to afatinib, suggesting that afatinib might also be effective in NSCLC patients with S768I mutations (9). The activity of afatinib in patients with uncommon EGFR mutations in the LUX-Lung clinical trials has been retrospectively reviewed (1). In this analysis, the uncommon mutation cohort was divided into three groups: point mutations or duplications in exons 18-21, de novo Thr790Met (T790M) mutations in exon 20, and insertion mutations in exon 20. Intriguingly, the best response to afatinib was noted in patients with point mutations or duplications in exons 18-21. In addition, Tanizaki et al. reported that afatinib was clinically effective for the treatment of NSCLC harboring EGFR mutations G719C and S768I, as well as KRAS E49K mutation (10). These findings encouraged us to initiate afatinib treatment in the present case (a patient with uncommon EGFR mutations), which led to remarkable tumor shrinkage and symptom improvement.

Afatinib-related adverse events included diarrhea, rash/ acne, stomatitis and nail effects. The diarrhea and rash/acne associated with afatinib monotherapy has been reported - in several clinical trials - to be more pronounced in comparison to first-generation EGFR-TKIs. Rash can have a negative impact on the quality of life, and cautious dermatology care is recommended. Likewise, TKI-induced diarrhea should be monitored carefully. When patients present with such severe toxicities, afatinib administration is interrupted and typically resumed at a reduced dose upon recovery. Yang et al. re- 


$\begin{array}{lllllll}d 1 & d 5 & d 10 & d 15 & d 20 & d 25 & d 30\end{array}$

\begin{tabular}{|c|c|c|c|}
\hline $\begin{array}{l}\text { Afatinib } \\
40 \mathrm{mg} / \mathrm{day}\end{array}$ & 30 & $30 \mathrm{mg} /$ day & $20 \mathrm{mg} /$ day \\
\hline
\end{tabular}

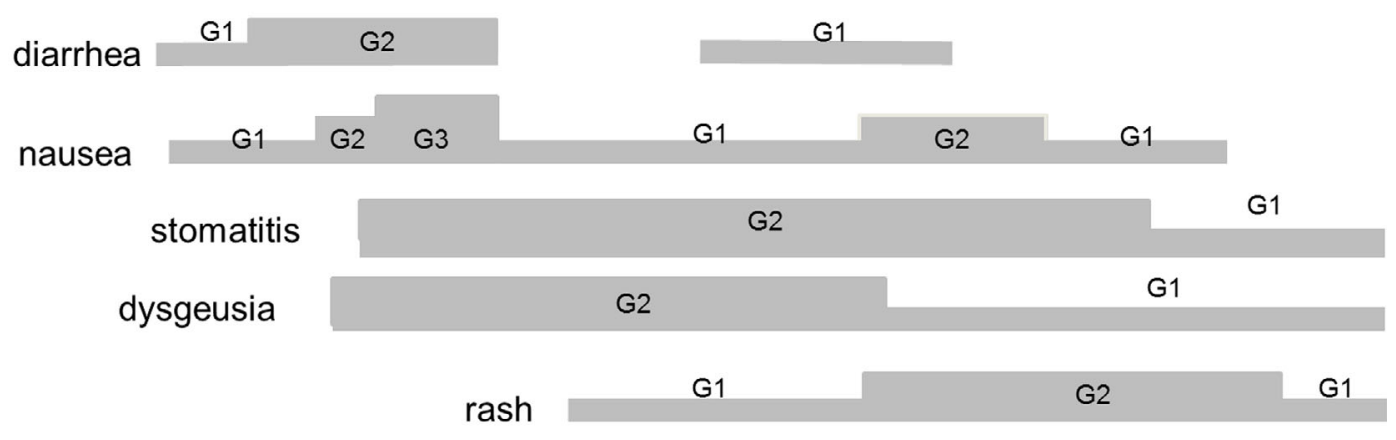

Figure 2. The clinical course of afatinib treatment. The dose of afatinib was reduced twice to relieve adverse clinical events. The patient currently receives afatinib $(20 \mathrm{mg} / \mathrm{day})$.

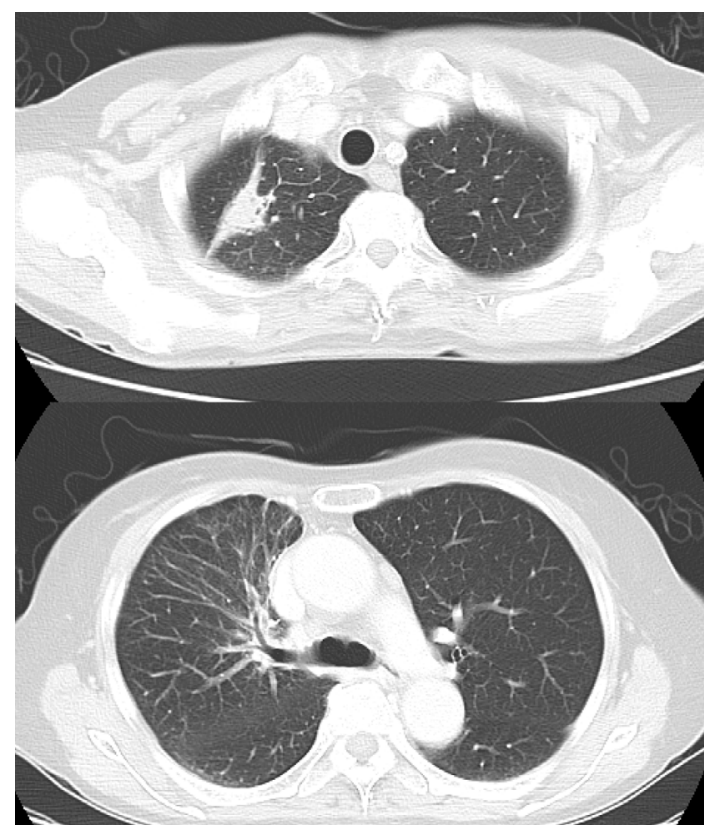

Figure 3. Chest computed tomography scans following afatinib treatment, showing the shrinkage of the primary lung tumor and the disappearance of carcinomatous lymphangitis.

ported that, in the LUX-Lung 3 and LUX-Lung 6 studies, the median PFS in patients in whom the dose was reduced during the first 6 months of afatinib treatment was similar to that of the patients in whom the dose was not reduced in the first 6 months of treatment (11.3 vs. 11.0 months, respectively) (11). Thus, the tolerability-guided dose adjustment of afatinib appears to reduce the incidence and severity of treatment-related adverse events without affecting efficacy. Afatinib dose management was similarly applied in the present case, and the patient has exhibited good response to afatinib (20 mg/day), without progression, for the last 12 months.
In conclusion, we herein reported the case of an NSCLC patient harboring rare complex G719X and S768I mutations, who responded to afatinib monotherapy. Based on these findings, second-generation EGFR-TKIs might be considered as an optimal treatment option for NSCLC cases harboring this type of EGFR mutation profile. A further analysis of the efficacy of EGFR-TKIs in such cases will lay the groundwork for developing optimized treatment protocols for NSCLC patients with uncommon EGFR mutations.

Author's disclosure of potential Conflicts of Interest (COI). Satoshi Oizumi: Honoraria, AstraZeneca and Eli Lilly.

\section{References}

1. Yang JC, Sequist LV, Geater SL, et al. Clinical activity of afatinib in patients with advanced non-small-cell lung cancer harbouring uncommon EGFR mutations: a combined post-hoc analysis of LUX-Lung 2, LUX-Lung 3, and LUX-Lung 6. Lancet Oncol 16: 830-838, 2015.

2. Chiu CH, Yang CT, Shih JY, et al. Epidermal growth factor receptor tyrosine kinase inhibitor treatment response in advanced lung adenocarcinomas with G719X/L861Q/S768I mutations. J Thorac Oncol 10: 793-799, 2015.

3. Wu SG, Chang YL, Hsu YC, et al. Good response to gefitinib in lung adenocarcinoma of complex epidermal growth factor receptor $(E G F R)$ mutations with the classical mutation pattern. Oncologist 13: 1276-1284, 2008.

4. De Pas T, Toffalorio F, Manzotti M, et al. Activity of epidermal growth factor receptor-tyrosine kinase inhibitors in patients with non-small cell lung cancer harboring rare epidermal growth factor receptor mutations. J Thorac Oncol 6: 1895-1901, 2011.

5. Wu JY, Yu CJ, Chang YC, et al. Effectiveness of tyrosine kinase inhibitors on "uncommon" epidermal growth factor receptor mutations of unknown clinical significance in non-small cell lung cancer. Clin Cancer Res 17: 3812-3821, 2011.

6. Watanabe S, Minegishi Y, Yoshizawa H, et al. Effectiveness of gefitinib against non-small-cell lung cancer with the uncommon 
EGFR mutations G719X and L861Q. J Thorac Oncol 9: 189-194, 2014.

7. Asahina H, Yamazaki K, Kinoshita I, et al. Non-responsiveness to gefitinib in a patient with lung adenocarcinoma having rare EGFR mutations S768I and V769L. Lung Cancer 54: 419-422, 2006.

8. Kobayashi Y, Togashi Y, Yatabe Y, et al. EGFR exon 18 mutations in lung cancer: molecular predictors of augmented sensitivity to afatinib or neratinib as compared with first- or third-generation TKIs. Clin Cancer Res 21: 5305-5313, 2015.

9. Banno E, Togashi Y, Nakamura Y, et al. Sensitivities to various epidermal growth factor receptor-tyrosine kinase inhibitors of uncommon epidermal growth factor receptor mutations L861Q and S768I: What is the optimal epidermal growth factor receptortyrosine kinase inhibitor? Cancer Sci 107: 1134-1140, 2016.
10. Tanizaki J, Banno E, Togashi Y, et al. Case report: durable response to afatinib in a patient with lung cancer harboring two uncommon mutations of EGFR and a KRAS mutation. Lung Cancer 101: 11-15, 2016.

11. Yang JC, Sequist LV, Zhou C, et al. Effect of dose adjustment on the safety and efficacy of afatinib for EGFR mutation-positive lung adenocarcinoma: post hoc analyses of the randomized LUXLung 3 and 6 trials. Ann Oncol 27: 2103-2110, 2016.

The Internal Medicine is an Open Access article distributed under the Creative Commons Attribution-NonCommercial-NoDerivatives 4.0 International License. To view the details of this license, please visit (https://creativecommons.org/licenses/ by-nc-nd/4.0/).

(C) 2018 The Japanese Society of Internal Medicine Intern Med 57: 993-996, 2018 